\title{
Self-Assembled 3-D Silicon Microscanners with Self-Assembled Electrostatic Drives
}

\author{
Richard R. A. Syms, Member IEEE
}

\begin{abstract}
Three-dimensional, electrostatically driven resonant torsion mirror microscanners are constructed by surface tension powered out-of-plane rotation of parts formed in bonded silicon-on-insulator. Simultaneous self-assembly of the fixed electrodes using a below-substrate limiter mechanism allows scanning perpendicular to the assembly axis, and direct drive allows high-Q operation.
\end{abstract}

Index Terms-MEMS, micromirror, microoptics, self-assembly.

$\mathbf{M}$ ICROOPTOELECTRO-MECHANICAL systems (MOEMS) are movable components constructed on $\mathrm{Si}$ substrates by surface micromachining to focus, filter, or deflect optical beams [1]. To process free-space beams, three-dimensional (3-D) structures are formed by out-of-plane rotation of parts hinged to the substrate [2]. Using these techniques, torsion mirror scanners with electrostatic [3] and electrothermal [4] drives have been demonstrated. In each case, an indirect drive was used, with the mirror driven via a link by an actuator on the substrate.

Similar components have been fabricated by surface tension self-assembly, a method of mass-parallel fabrication of 3-D microstructures [5]. Rotation is powered by melting small pads of material (solder, glass, or photoresist) linking movable parts to the substrate. Recent work has used thick resist, with single crystal parts formed in bonded silicon-on-insulator (BSOI). Assembly has involved $45^{\circ}$ rotation, with the final geometry fixed by a limiter [6].

Fig. 1(a) shows the mechanism used, which involves simultaneous rotation of two parts (FRAME-L and FRAME-R) in opposite directions. Catches on the parts prevent further motion when each has rotated through $45^{\circ}$. The assembly accuracy is extremely high, but the mechanism is bulky and, hence, most suitable for major component frames.

This two-part structure has been used in electrostatically driven torsion mirror scanners [6]. FRAME-R carried a mirror mounted on a torsion bar with its axis parallel to the substrate. The mirror carried one half of a comb electrostatic drive, while the other half was attached to the substrate. After assembly, the mirror could be excited into resonant torsional oscillation by a harmonic electrostatic force between the two halves of the drive. Because of the direct nature of the drive, quality factors as high as 60 were demonstrated.

Manuscript received April 25, 2000; revised July 7, 2000. This work was supported by EPSRC.

The author is with the Department of Electrical and Electronic Engineering, Imperial College, Exhibition Road, London, SW7 2BT, U.K. (e-mail r.syms@ic.ac.uk).

Publisher Item Identifier S 1041-1135(00)09600-2.

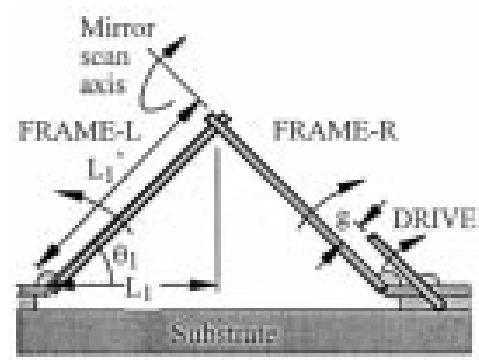

(a)

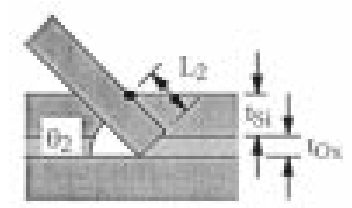

(b)

Fig. 1. (a) Surface tension self-assembly using above- and below-substrate mechanical limiters; (b) detail of below-substrate limiter.

The design had several limitations. First, the skewed electrode layout resulted in a high drive voltage, because of the weak electrostatic field. Second, there was a trade off between the voltage and the scan angle, because a reduction in separation between the halves of the comb could only be performed by lengthening the moving electrode fingers, reducing the angle of turn before they struck the substrate. Finally, only mirror axes parallel to the substrate could be used, to avoid the electrodes clashing.

In this paper, we show how to overcome these difficulties by further self-assembly operations, and introduce a new mechanism that allows parts as small as the fixed half of the electrode to be reconfigured. Fig. 1(a) and (b) shows the mechanism, which is based on two cranks attached to a third movable part DRIVE near its hinge. Motion is prevented when the cranks reach the substrate. The accuracy of this mechanism is inherently low, as we show below, but it is sufficient for sub-component assembly. Here, we show how it can be used to construct a mirror scanner with a rotation axis orthogonal to that of earlier devices [6], driven by an electrostatic drive that is reconfigured into a staggered comb.

Fig. 2 shows the layout of the scanner before assembly. There are three moving parts: the left- and right-hand halves of the frame, and the fixed half of the drive. The parts are constructed by deep reactive ion etching (DRIE) through the bonded Si layer of a BSOI wafer, using an inductively coupled plasma etcher. This layer had a nominal thickness of $t_{\mathrm{Si}}=5 \mu \mathrm{m}$, which ranged in practice up to $5.5 \mu \mathrm{m}$. The movable parts are perforated, to allow removal of a sacrificial $\mathrm{SiO}_{2}$ layer of thickness $t_{\mathrm{Ox}}=$ 


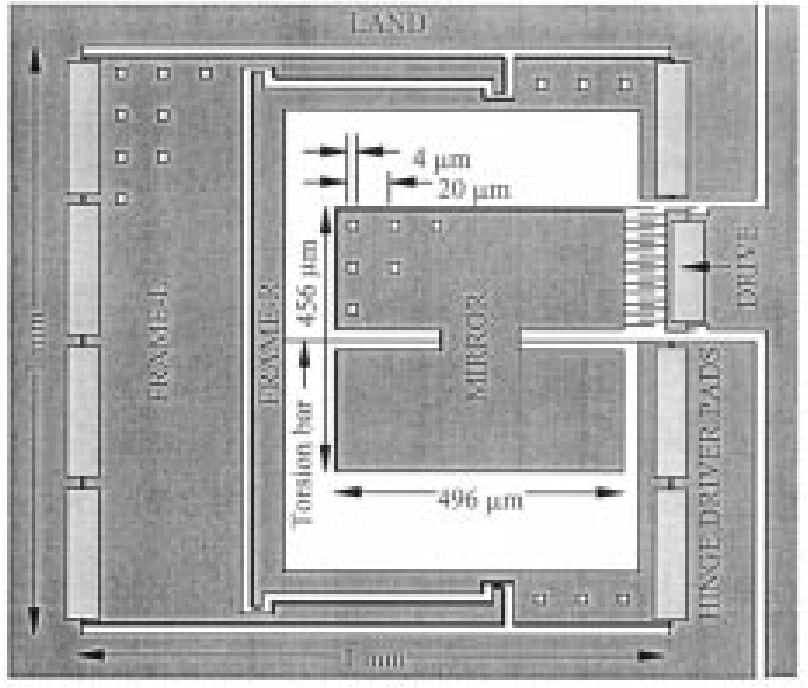

Fig. 2. Layout of torsion mirror scanner with self-assembling electrostatic drive.

$2 \mu \mathrm{m}$ using BHF. The fixed and moving parts are linked by $250 \times 40-\mu \mathrm{m}$ pads of Hoechst AZ4562 resist with a thickness of $\approx 12 \mu \mathrm{m}$. FRAME-R carries a $496 \mu \mathrm{m} \times 456-\mu \mathrm{m}$ mirror on a 6- $\mu \mathrm{m}$-wide torsion bar, which is now orthogonal to the assembly axes. DRIVE carries 13 electrode fingers, each $6 \mu \mathrm{m}$ wide and $75 \mu \mathrm{m}$ long, separated from the moving fingers by $2-\mu \mathrm{m}$-wide lateral gaps.

The important frame dimensions $L_{1}$ and $L_{1}^{\prime}$ are $500 \mu \mathrm{m}$ and $500 \sqrt{ } 2 \mu \mathrm{m}$, respectively. The key crank dimension $L_{2}$ was chosen by noting that contact is just made to the substrate when $\theta_{2}=45^{\circ}$ if $L_{2}=t_{\mathrm{Si}}$ and $\sqrt{ } 2 t_{\mathrm{Si}}=t_{\mathrm{Si}}+t_{\mathrm{Ox}}$, so that $t_{\mathrm{Si}}=t_{\mathrm{Ox}} /\{\sqrt{ } 2-1\}$. This value corresponds to $t_{\mathrm{Si}}=4.83 \mu \mathrm{m}$ when $t_{\mathrm{Ox}}=2 \mu \mathrm{m}$, slightly lower than the $5-\mu \mathrm{m}$ nominal value. $L_{2}$ was therefore taken as $5 \mu \mathrm{m}$ to make contact just before $\theta_{2}=45^{\circ}$. The assembly axis of DRIVE is placed slightly to the right of that of FRAME-R, so that the fixed electrode will eventually stand a distance $g$ in front of the moving electrode. The relative position of the two electrodes may now be adjusted by altering this separation, without lengthening the moving fingers. Here, the initial separation is $21.2 \mu \mathrm{m}$, so that $g=\{21.2 / \sqrt{ } 2-5\}=10 \mu \mathrm{m}$.

Assembly is performed by melting the resist at $145{ }^{\circ} \mathrm{C}$. Surface tension torque causes FRAME-L and FRAME-R to counter-rotate until the above-substrate mechanism catches. DRIVE rotates in the same direction as FRAME-R until the below-substrate limiter engages. A realistic yield $(>50 \%)$ was obtained. The frames typically assembled accurately, and optical measurements showed angular errors as low as 2 arc min in constructing $45^{\circ}$ planes. This error is less than corresponding values of $\approx 1^{\circ}$ presented earlier [6], and the improvement is attributed to the use of DRIE instead of RIE to define the parts. However, SEM inspection of self-assembled electrodes showed much larger errors. A number over-rotated, to a final angle of $90^{\circ}$ or more, while the majority stabilized at angles up to $10^{\circ}$ below $45^{\circ}$. Fig. 3(a) and (b) shows the overall structure and drive of a correctly assembled device, showing the fixed electrode in front of the moving electrode.
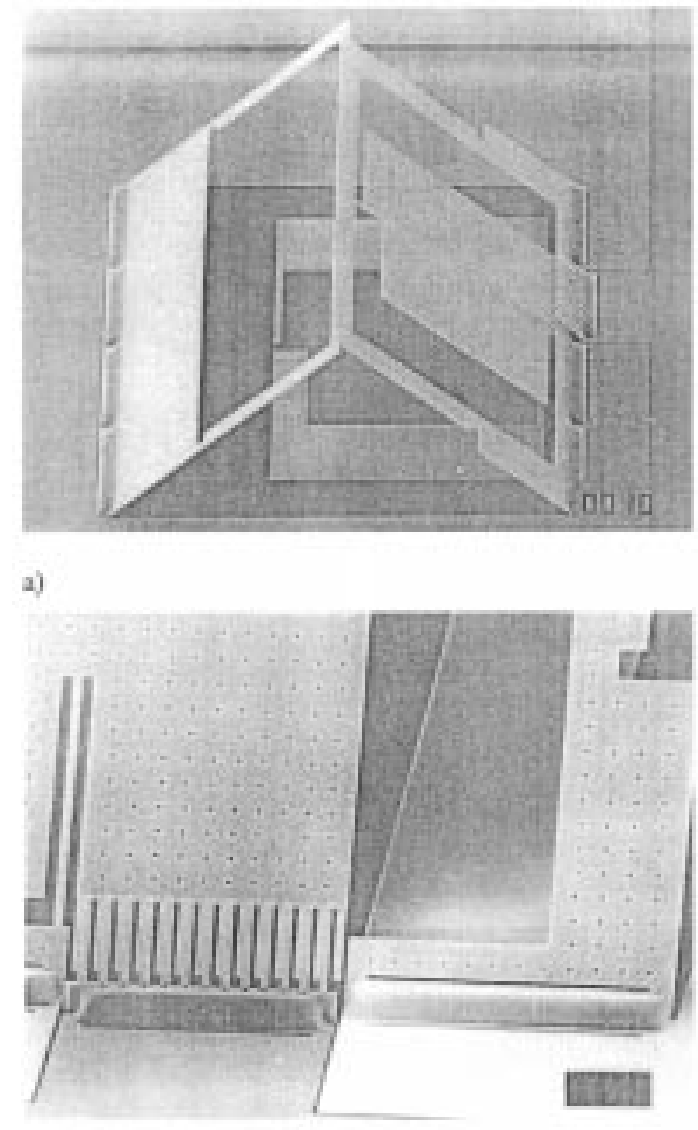

b)

Fig. 3. SEM views of (a) assembled scanner and (b) self-assembled drive.

The difference in assembly accuracy of the two assembly mechanisms may be explained in terms of their sensitivity to dimensional errors. We first consider the main frame. By geometry, we may obtain $\theta_{1}$ in Fig. 1(a) as $L_{1}=L_{1}^{\prime} \cos \left(\theta_{1}\right)$. For $45^{\circ}$ rotation, we require $L_{1}^{\prime}=L_{1} \sqrt{ } 2$. These dimensions will be altered by erosion during processing. However, not all features are affected equally. Uniform erosion mainly affects features defined by etched planes with anti-parallel orientations (e.g., trenches); dimensions determined by parallel planes (e.g., the separation between treads in an etched staircase pattern) are relatively unaffected. Here, the planes determining $L_{1}^{\prime}$ are parallel, while those defining $L_{1}$ are anti-parallel, so the dimensional uncertainty lies mainly in $L_{1}$. The angular error $\delta \theta_{1}$ caused by a variation $\delta L_{1}$ in $L_{1}$ is then $\delta \theta_{1}=-\delta L_{1} / L_{1}$. For $L_{1}=500$ $\mu \mathrm{m}, \delta \theta_{1}=2^{\prime}$ corresponds to $\delta L_{1}=0.3 \mu \mathrm{m}$. This error is comparable to the thickness of resist used to define the parts $(0.35$ $\mu \mathrm{m})$, suggesting that accuracy might be increased by improving lithography and etching, or by increasing the absolute size of the structure.

We now perform a similar analysis for the self-assembled electrode. The dimension $L_{2}$ is determined by parallel etched planes, and (although small) may be considered well defined. Errors such as complete over-rotation and slight under-rotation are traceable to variations in the bonded silicon thickness. For example, over-rotation implies that the crank has not engaged with the substrate at all; this will occur if the crank radius is less 


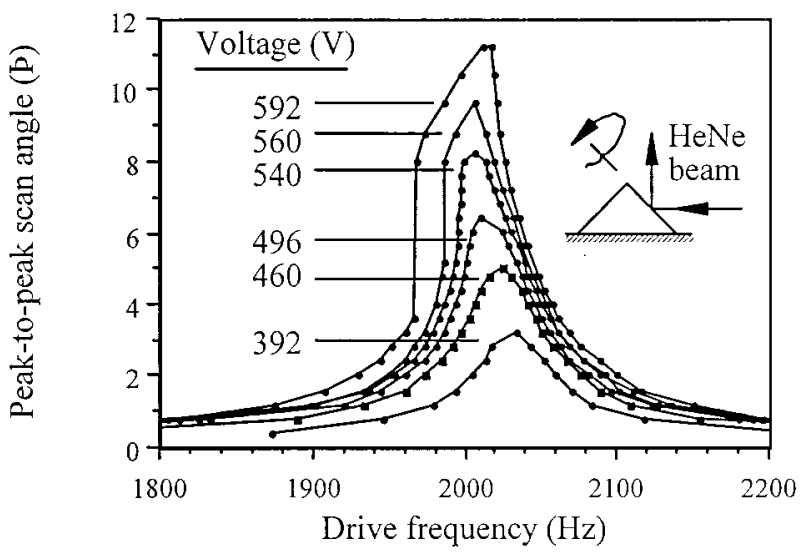

Fig. 4. Variation of optical scan angle with frequency, for different drive voltages.

than the total layer thickness, so that $\sqrt{ }\left(L_{2}^{2}+t_{\mathrm{Si}}^{2}\right)<t_{\mathrm{Si}}+t_{\mathrm{Ox}}$ Values of $t_{\mathrm{Si}}$ above $\left(L_{2}^{2}-t_{\mathrm{Ox}}^{2}\right) / 2 t_{\mathrm{Ox}}=5.25 \mu \mathrm{m}$ fall into this category. Lower values of $t_{\mathrm{Si}}$ allow the crank to engage, but yield too small a final angle. A minimum angular error of $\delta \theta_{2}=$ $-1.4^{\circ}$ is obtained when $t_{\mathrm{Si}}$ is just below $5.25 \mu \mathrm{m}$, and the error rises to $\delta \theta_{2}=-8.1^{\circ}$ when $t_{\mathrm{Si}}=5.00 \mu \mathrm{m}$. These results suggest that the spread in the bonded silicon thickness is responsible for the observed effects, and that the angular errors obtained are relatively large because of the small radius of the crank.

After assembly, scanners are sputter-coated with $500 \AA$ Al. Performance is assessed by measuring the deflection of a $\mathrm{HeNe}$ laser beam when a sinusoidal voltage is applied between DRIVE and the surrounding LAND. Variations in the angle of the self-assembled electrode do not prevent operation, but cause changes in drive voltage. Fig. 4 shows a typical variation of peak-to-peak optical scan angle with electrical frequency, for different peak-to-peak voltages. A quality factor of $\approx 45$ is obtained for small scans, and a scan angle of $\approx 11^{\circ}$ is obtained at $\approx 600 \mathrm{~V}$. At this point, the peak mirror deflection is $\approx 2.75^{\circ}$, and the outer moving electrodes are just passing between the fixed ones. As the scan increases, the resonant frequency reduces slightly, and the resonance loses symmetry. These features are characteristic of a nonlinear softening suspension.

In conclusion, we have demonstrated the use of photoresist-powered surface tension self-assembly and bonded silicon-on-insulator to form a complex torsion mirror microscanner driven by a staggered electrostatic comb drive. A new mechanism based on below-substrate mechanical limiting has been developed to reconfigure the drive and, hence, allow a mirror turn axis perpendicular to that of earlier self-assembled scanners. The accuracy of this mechanism has been shown to depend mainly on dimensional control of the BSOI material.

\section{ACKNOWLEDGMENT}

The author is very grateful to BCO Technologies (NI) Ltd. for manufacturing the BSOI material and performing the ICP etching.

\section{REFERENCES}

[1] R. S. Muller and Y. K. Lau, "Surface micromachined microoptical elements and systems," Proc. IEEE, vol. 86, pp. 1705-1720, Aug. 1998.

[2] K. S. Pister, M. W. Judy, S. R. Burgett, and R. S. Fearing, "Microfabricated hinges," Sensors Actuators, vol. A33, pp. 249-256, 1992.

[3] M.-H. Kiang, O. Solgaard, and K. Y. Lau, "Electrostatic combdrive-actuated micromirrors for laser-beam scanning and positioning," J. Micromech. Syst., vol. 7, pp. 27-37, 1998.

[4] J. T. Butler, V. M. Bright, and J. R. Reid, "Scanning and rotating micromirrors using thermal actuators," Proc. SPIE, vol. 3131, pp. 134-144, 1997.

[5] R. R. A. Syms and E. M. Yeatman, "Self-assembly of fully three-dimensional micro-structures using rotation by surface tension forces," Electron. Lett., vol. 29, pp. 662-664, 1993.

[6] R. R. A. Syms, "Surface tension powered self-assembly of 3-D micro-optomechanical structures," IEEE/ASME J. Microelectromech. Syst., vol. 8, pp. 448-455, 1999. 